\title{
COMBINATORIAL TOPOLOGY OF AN ANALYTIC FUNCTION ON THE BOUNDARY OF A DISK
}

\author{
BY CHARLES J. TITUS
}

Communicated by Edwin Moise, August 12, 1960

Preliminaries. A complex valued function $\zeta(t)$ defined on an oriented circle $S$ of circumference $c, t$ the usual distance parameter, $0 \leqq t<c$, is a regular representation if it possesses a continuous nonvanishing derivative $\zeta^{\prime}(t)$. An image point $\zeta_{0}$ is a simple crossing point if there exist exactly two distinct numbers $t_{0}^{\prime}$ and $t_{0}^{\prime \prime}$ such that $\zeta\left(t_{0}^{\prime}\right)=\zeta\left(t_{0}^{\prime \prime}\right)=\zeta_{0}$ and if the tangents $\zeta^{\prime}\left(t_{0}^{\prime}\right)$ and $\zeta^{\prime}\left(t_{0}^{\prime \prime}\right)$ are linearly independent. A regular representation is normal (Whitney) if it has a finite number of simple crossing points and has for every other image point $\zeta$ but one preimage point $t$. A pair of representations $\tilde{\zeta}$ and $\zeta$ are topologically equivalent if there exists a sense-preserving homeomorphism $h$ of $S$ onto $S$ such that $\tilde{\zeta}=\zeta \circ h$.

A mapping $F$ of a disk $D,|z|<R$, is open if, for every open set $U$ in $D, F(U)$ is open in the plane; $F$ is light if the preimage of each image point is totally disconnected; $F$ is properly interior on $\bar{D}$, $|z| \leqq R$, if $F$ is continuous on $\bar{D}, F \mid$ bdy $D$ is locally topological, $F$ is sense-preserving, light and open on $D$. It can be shown (using results of Carathéodory, Stoilow, Whyburn) that given a properly interior mapping $F$ there exists an analytic function $W$ on $D$ that is locally topological near and on bdy $D$ and there exists a sense-preserving homeomorphism $H$ of $\bar{D}$ onto $\bar{D}$ such that $F=W \circ H$.

A representation $\zeta$ will be called an interior boundary [analytic boundary] if $\zeta$ is locally topological and if there exists a properly interior mapping $F$ [an analytic function $W$ that is locally topological near and on bdy $D]$ such that $F\left(R e^{i t}\right) \equiv \zeta(t)\left[W\left(R e^{i t}\right) \equiv \zeta(t)\right]$. Thus, every interior boundary is topologically equivalent to an analytic boundary.

The problem probably first arose in the study of the SchwartzChristoffel mapping function (Schwartz, Schlaefli, Picard) and, in this context, was formulated essentially as follows.

Let $Z_{0}, Z_{1}, \cdots, Z_{n-1}$ be a sequence of $n$-distinct complex numbers which are in general position. By connecting these points consecutively from $Z_{k}$ to $Z_{k+1}$, $\bmod n$, a closed oriented polygon is formed. Let $\alpha_{k} \pi$ be the angle from $Z_{k}-Z_{k-1}$ to $Z_{k+1}-Z_{k}$ with $-1<\alpha_{k}<1$. Then for any set of $n$ real number and any complex number $A \neq 0$ the function 


$$
\Phi(Z)=A\left(Z-a_{0}\right)^{-\alpha_{0}}\left(Z-a_{1}\right)^{-\alpha_{1}} \cdots\left(Z-a_{n-1}\right)^{-\alpha_{n-1}},
$$

with $-\pi / 2<\arg \left(Z-\alpha_{k}\right)<\pi / 2$, is an analytic function on the upper half plane; furthermore

$$
W(z)=\int_{i}^{z} \Phi(Z) d Z+B
$$

is also analytic there and maps the real axis onto a possibly different polygon with $W\left(a_{k}\right)=Z_{k}^{\prime}$ but with $Z_{k}^{\prime}-Z_{k-1}^{\prime}$ having the same direction as $Z_{k}-Z_{k-1}$.

Problem A (Emile Picard, Traité d'analyse, vol. 2, p. 313). Find necessary and sufficient conditions on $Z_{0}, Z_{1}, \cdots, Z_{n-1}$ so that there exist complex numbers $A, B$ and real numbers $a_{0}, a_{1}, \cdots, a_{n-1}$ so that $W\left(a_{k}\right)=Z_{k}$, and thus that the real axis is mapped onto the polygon determined by the given $Z_{k}$. (Actually Schlaefli and others were concerned also with the problem of finding an effective method for determining the $a_{k}$.)

Some time ago a clearly related problem was formulated by Loewner (circa 1948) which will be stated in the form:

Problem B (Charles Loewner). Given a normal representation $\zeta$ of a closed curve find necessary and sufficient conditions that $\zeta$ be equivalent to an analytic boundary (or, what is the same thing, that $\zeta$ be an interior boundary).

Problem A is a corollary of Problem B. In this paper a solution to Problem B is announced. More precisely Problem A was concerned only with oriented polygons with a tangent winding number of one.

STATEMENT OF RESUlts. In the following $\zeta$ will always be a normal representation; the simple crossing points will be called vertices. Let $\tau[\zeta]$ be the tangent winding number of $\zeta$ and let $\omega(\zeta, \pi)$ be the winding number (index) of $\zeta$ about a point $\pi, \pi \in[\zeta]$. The outer boundary of $\zeta$ is the subset of $[\zeta]$ which is contained in the closure of the un-

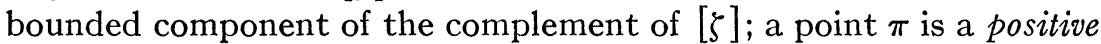
outer point if $\pi$ is on the outer boundary and is not a vertex and if there exist points $\pi^{\prime}$ arbitrarily close to $\pi$ such that $\omega\left(\zeta, \pi^{\prime}\right)=+1$.

Lemma. If $\zeta$ is an interior boundary then $\tau[\zeta] \geqq 1$ and $\omega(\zeta, \pi) \geqq 0$ for all $\pi \in[\zeta]$.

Because of this Lemma only curves $\zeta$ which satisfy these conditions need be considered; call this class $C^{+}$.

Begin at a positive outer point $\pi=\zeta(0)$ and traverse the curve in the direction of its sense. Index the vertices using consecutively the integers from 0 to $n-1, \zeta_{0}, \zeta_{1}, \cdots, \zeta_{n-1}$. Let the $2 n$ preimages of the 
vertices be denoted by $s_{k}$ and index so that $0<s_{0}<s_{1}<\cdots<s_{2 n-1}$ $<c$. If $\zeta\left(s_{j}\right)=\zeta\left(s_{k}\right), s_{j} \neq s_{k}, s_{j}$ is also denoted by $s_{\mathbf{k}}^{*}$ (and $s_{k}$ by $s_{j}^{*}$ ). Let $\nu_{k}$ be defined, with $\zeta(t)=\xi(t)+i \eta(t)$, by

$$
\nu_{k}=\nu\left(s_{k}\right)=\operatorname{sgn}\left|\begin{array}{cc}
\xi^{\prime}\left(s_{k}^{*}\right) & \eta^{\prime}\left(s_{k}^{*}\right) \\
\xi^{\prime}\left(s_{k}\right) & \eta^{\prime}\left(s_{k}\right)
\end{array}\right| .
$$

If the sequence $\left\{s_{k}\right\}$ together with the * operation, the $\nu_{k}$ and the fact that $\zeta(0)=\pi$ is a positive outer point are given then the oriented curve represented by $\zeta$ is determined up to a sense preserving homeomorphism of the plane onto itself (follows from e.g. Adkisson and MacLane and Gehman). See Figure 1 in which

$$
\begin{gathered}
\nu_{0}=\nu_{1}=\nu_{3}=\nu_{5}=1, \quad \nu_{2}=\nu_{4}=\nu_{6}=\nu_{7}=-1: \\
s_{0}^{*}=s_{7}, \quad s_{1}^{*}=s_{6}, \quad s_{2}^{*}=s_{3}, \quad s_{4}^{*}=s_{5} .
\end{gathered}
$$

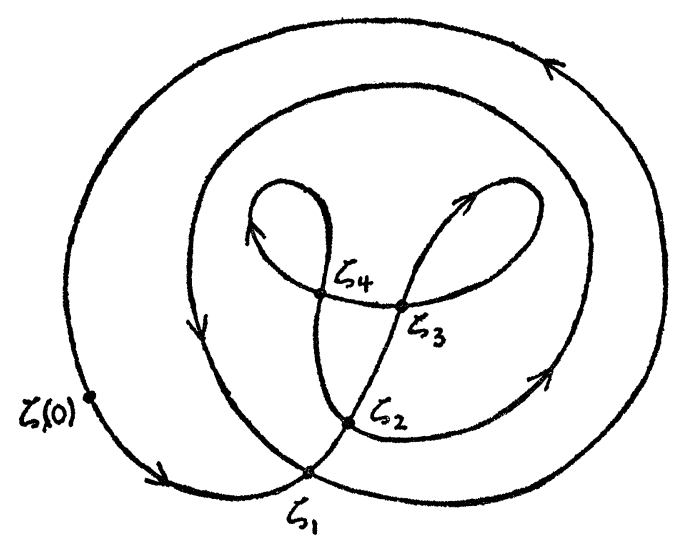

FIG. 1

Select any point $\pi$ on the outer boundary of $\zeta, \zeta(0)=\pi, \zeta \in C^{+}$and let $s_{k}$ be the number with the smallest index so that $\nu_{k}=-1$. At least one of the following situations must arise:

CASE I. $s_{k}^{*}<s_{k}$.

CASE II. $s_{k}^{*}>s_{k}$.

In the later case for each choice of an $s_{j}<s_{k}$ there corresponds one of the two situations $\mathrm{II}^{\prime}$ and $\mathrm{II}^{\prime \prime}$ :

CASE II'. $s_{k}^{*}>s_{k}$ and $s_{j}<s_{k}<s_{k}^{*}<s_{j}^{*}$,

CASE II". $s_{k}^{*}>s_{k}$ and $s_{j}<s_{k}<s_{j}^{*}<s_{k}^{*}$.

In each of these situations a cut is defined that breaks $\zeta$ up into a pair of piecewise regular representations $\zeta^{*}$ and $\zeta^{* *}$. (It turns out 
that $\zeta^{*}$ and $\zeta^{* *}$ can be smoothed and altered slightly so that each becomes a normal representation. This step, which is bothersome and simple technically, will be omitted here; in what follows the reader may ignore this problem and pretend that $\zeta^{*}$ and $\zeta^{* *}$ have already been made normal.)

In Case I define on circles of circumference $c^{*}$ and $c^{* *}$ :

$$
\begin{aligned}
\zeta^{*}(t) & =\zeta\left(t+s_{k}^{*}\right), \\
\zeta^{* *}(t) & = \begin{cases}\zeta(t), & 0 \leqq t \leqq s_{k}-s_{k}^{*}=c^{*} ; \\
\zeta\left(s_{k}-s_{k}^{*}+t\right), & s_{k}^{*} \leqq t \leqq c-s_{k}+s_{k}^{*}=c^{* *} .\end{cases}
\end{aligned}
$$

In Case II select $s_{j}<s_{k}$ and if $s_{j}<s_{k}<s_{\mathbf{k}}^{*}<s_{j}^{*}$ we have Case II'; define on circles of circumference $c^{*}$ and $c^{* *}$ :

$$
\begin{aligned}
& \zeta^{*}(t)= \begin{cases}\zeta\left(t+s_{j}\right), & 0 \leqq t \leqq s_{k}-s_{j}, \\
\zeta\left(s_{j}+s_{k}-s_{k}^{*}+t\right), & s_{k}-s_{j} \leqq t \leqq s_{k}-s_{j}+s_{j}^{*}-s_{k}^{*}=c^{*} ;\end{cases} \\
& \zeta^{* *}(t)= \begin{cases}\zeta(t), & 0 \leqq t \leqq s_{k}^{*}, \\
\zeta\left(s_{k}-s_{k}^{*}-t\right), & s_{k}^{*} \leqq t \leqq s_{k}^{*}+s_{k}-s_{j}, \\
\zeta\left(s_{j}+s_{j}^{*}-s_{k}-s_{k}^{*}+t\right), & s_{k}^{*}+s_{k}-s_{j} \leqq t \leqq c+s_{j}+s_{j}^{*}-s_{k}-s_{k}^{*}=c^{* *} ;\end{cases}
\end{aligned}
$$

but if in Case II, $s_{j}<s_{k}<s_{j}^{*}<s_{\boldsymbol{k}}^{*}$, one has Case II" and define

$$
\begin{aligned}
& \zeta^{*}(t)= \begin{cases}\zeta\left(s_{j}^{*}+t\right), & 0 \leqq t \leqq s_{k}^{*}-s_{j}^{*}, \\
\zeta\left(s_{j}^{*}+s_{k}^{*}+s_{k}-t\right), & s_{k}^{*}-s_{j}^{*} \leqq t \leqq s_{k}+s_{j}+s_{k}^{*}-s_{j}^{*}=c^{*} ;\end{cases} \\
& \zeta^{* *}(t)= \begin{cases}\zeta(t), & 0 \leqq t \leqq s_{j}^{*}, \\
\zeta\left(s_{j}-s_{j}^{*}+t\right), & s_{j}^{*} \leqq t \leqq s_{j}^{*}-s_{j}+s_{k}, \\
\zeta\left(s_{j}-s_{j}^{*}+s_{k}^{*}-s_{k}+t\right), & s_{j}^{*}-s_{j}+s_{k} \leqq t \leqq c+s_{j}^{*}-s_{j}+s_{k}-s_{k}^{*}=c^{* *} .\end{cases}
\end{aligned}
$$

These three cuts are illustrated in Figures 2, 3 and 4.

Assuming the $\zeta^{*}$ and $\zeta^{* *}$ altered so that they are normal (as commented upon parenthetically above) the cut process can be continued so long as the new representations remain in $\mathrm{C}^{+}$. A normal representation $\zeta$ possesses a complete cut sequence provided that the representations generated by successive cuts always remain in $\mathrm{C}^{+}$; thus ultimately the representations (in the slightly altered form) describe simple closed positively oriented curves. 


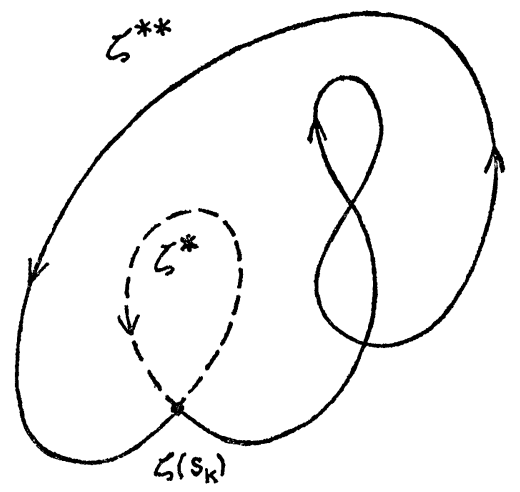

FIG. 2
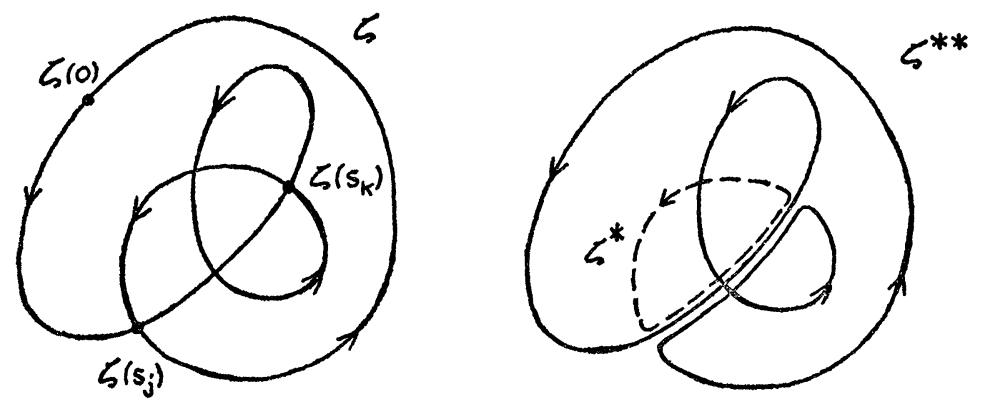

FIG. 3
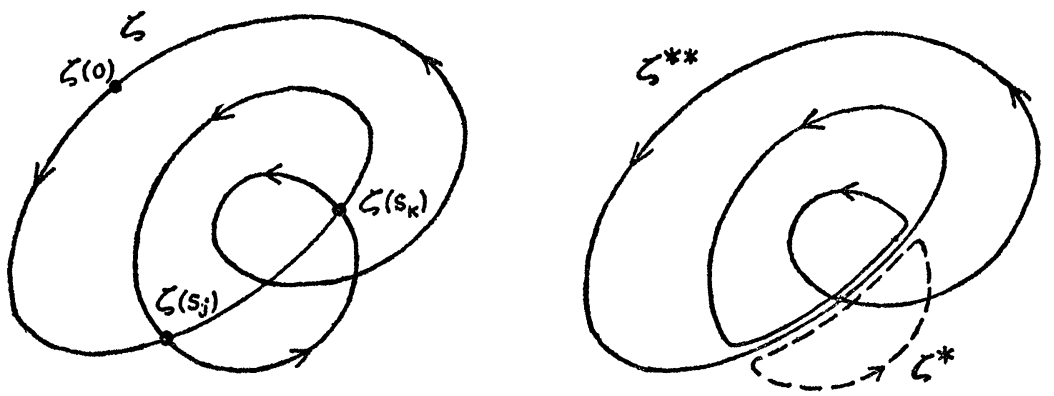

FIG. 4

Principal Lemma. A normal representation $\zeta$ is an interior boundary if and only if (i) there exists a cut of type I and the corresponding $\zeta^{*}$ and $\zeta^{* *}$ are both interior boundaries, or (ii) there does not exist a cut of type I (whence there must exist cuts of type II) but there exists an $s_{j}$ and a corresponding cut of type $\mathrm{II}^{\prime}$ or $\mathrm{II}^{\prime \prime}$ so that $\zeta^{*}$ and $\zeta^{* *}$ are interior boundaries. 
It is also true that the slightly altered $\zeta^{*}$ and $\zeta^{* *}$ have strictly less vertices than the original $\zeta$.

It follows directly from this Lemma that

THEOREM 1. A normal representation $\zeta$ is an interior boundary if and only if $\zeta$ possesses a complete cut sequence.

Let $\mu$ be the number of cuts of type I required in a complete cut sequence for a given interior boundary $\zeta$.

THEOREM 2. If $W$ is an analytic function which extends a representation equivalent to $\zeta$ to the disk then $W(z)$ has precisely $\mu$ zeros (counting multiplicity) in the disk, (thus e.g., $\tau[\zeta]=\mu+1$ ).

COROLLARY. $\zeta$ has a complete cut sequence with $\mu=0$ (no cuts of type I) if and only if there is a sense-preserving local homeomorphism $F$ which extends $\zeta$ to the disk.

\section{BiBLIOGRAPHY}

1. V. W. Adkisson and Saunders MacLane, Extensions of homeomorphisms on the sphere, Lectures in Topology, University of Michigan Press, 1941, pp. 223-235.

2. Constantin Carathéodory, Conformal representation, Cambridge University Press, 1932.

3. H. M. Gehman, On extending a continuous 1-1 correspondence of two plane continuous curves to a correspondence of their planes, Trans. Amer. Math. Soc. vol. 42 (1936) pp. 79-86.

4. Emile Picard, Traité d'analyse (2), pp. 310-314.

5. Simion Stoilow, Leçons sur les principes topologiques de la théorie des fonctions analytiques, Paris, Gauthier-Villars, 1956.

6. C. J. Titus, $A$ theory of normal curves and some applications, Pacific J. Math. vol. 10 (1960) pp. 1083-1096.

7. - The image of the boundary under a local homeomorphism, Functions of a Complex Variable, University of Michigan Press, 1955, pp. 433-435.

8. Hassler Whitney, On regular closed curves in the plane, Compositio Math. vol. 4 (1937) pp. 276-284.

9. G. T. Whyburn, Topological analysis, Princeton University Press, 1958.

UNIVERSITY OF Michigan 